\title{
DESIGN AND EVALUATION OF ADAPTIVE COLLISION AVOIDANCE SYSTEMS
}

\author{
Husam Muslim ${ }^{1}$, Makoto Itoh ${ }^{2}$ \\ ${ }^{1}$ Graduate School of Systems and Information Engineering, Risk Engineering Department \\ ${ }^{2}$ Faculty of Engineering, Information and Systems \\ University of Tsukuba, Tsukuba, Ibaraki, Japan \\ Email: hussam@css.risk.tsukuba.ac.jp
}

\begin{abstract}
Summary: Taking a human factors approach, the present study aims at improving driver interaction with automation by improving driver trust in and understanding of the system and enhancing system design. First, a driving experiment was conducted to investigate how driver understanding of the system capabilities effects driver performance and trust. The experiment compared two driver assistance systems for avoiding collisions during critical lane change: one was a haptic steering control that manipulates the steering wheel friction torque, and the other was an automatic steering control that decouples the driver during critical conditions. The results indicate that, especially in critical situations when driver expectation of the system and system capabilities were not aligned, the driversystem interaction was significantly affected by the way control is allocated between agents. To improve system design in terms of functional allocation and capabilities, the study proposes an enhanced adaptive collision avoidance system in which control is allocated dynamically depending on the situation. This system was assessed in a second driving experiment. While the diver-system interactions significantly improved compared to the haptic and automatic steering control systems, in terms of safety, it did not perform as well as expected. A third experiment, using long term simulator training, was conducted to enhance drivers' understanding of and trust in the system. The training interaction revealed that drivers adapted more easily to the system, improving driver performance, system effectiveness, and safety. The findings highlight how user training can improve human-automation interaction.
\end{abstract}

\section{INTRODUCTION}

Automation has been applied in all aspects of human-machine systems including car driving. Advances in automotive automation systems have long been used to improve traffic systems, task performance, workload, and safety (Carsten et al., 2012). When employed in safety-critical conditions, the benefits of automation assistance in avoiding collisions and reducing human errors have been demonstrated (Kusano and Gabler, 2012). However, human errors in situations involving automation assistance have also been reported (Parasuraman and Riley, 1997). These situations are most likely to occur due to conflicts in goals and actions of the interacting agents, or due to lack of human understanding of system capabilities and limitations (*Muslim and Itoh, 2018). Such conflicts and lack of understanding can result in inappropriate interactions between humans and automation (Parasuraman and Riley, 1997). How a human interacts with automation is an important factor to determine task performance, system effectiveness, and safety (Hoc, 2000). 
Previous research has indicated that humans can easily understand, accept and adapt to a system as long as they are maintained as the final authority over the system (Abbink et al., 2012; Itoh and Inagaki, 2014; Muslim and Itoh, 2017). However, other researchers have observed that when maintained as the final authority, they may, in some highly critical situations, choose to ignore or override the system (**Muslim and Itoh, 2018; Sheridan and Parasuraman, 2006). This contradictions suggest the need for adaptive automation that determines how much control is to be allocated to the human based on the situation. A challenging task is determining the appropriate automation assistance for the given situation depending on risk factors and situation handling ability. For such a system, it is necessary to understand how humans interact with the different levels of automation and authority and how this effects system effectiveness. Some pitfalls could be expected as the human may not be easily able to deal with dynamically changing configurations of adaptive control systems (Parasuraman and Riley, 1997).

Using a driving simulator, the present study conducted three sequential experiments to investigate the effects of automation authority and control allocation on drivers' understanding of the adaptive system in safety-critical situations. The second aim was to design an effective and usable adaptive collision avoidance system in which the control can be flexibly and dynamically transferred between agents depending on capabilities and limitations of both agents. The third aim was to enhance human-automation interaction using training approach.

\section{METHODOLOGY}

Three experiments with a total of 130 participants were conducted, as shown in Table 1. All participants were daily drivers with a valid Japanese driving license. The driving simulator setup emulates a real Honda car with a single adjustable car seat, a motorized steering wheel, and an automatic transmission system (Figure 1). The driver scene was projected on a curved screen creating 120 degrees field of view with three small LCD displays to simulate the side and rear views. However, with the available field of view, the drivers were unable to see the blind spot in the adjacent lane. This was to encourage drivers to build a mental model of the assistance systems and experience the benefits of the system in collision avoidance when changing lanes. All drives were conducted on two-lane highway where the speed limit was set to $80 \mathrm{~km} / \mathrm{h}$. The drivers were instructed to drive safely in the left-hand lane. Slower vehicles $(70 \mathrm{~km} / \mathrm{h})$ were placed randomly on the driving course to induce lane change maneuvers. To simulate hazardous lane changes, vehicles were placed differently in the cruising lane.

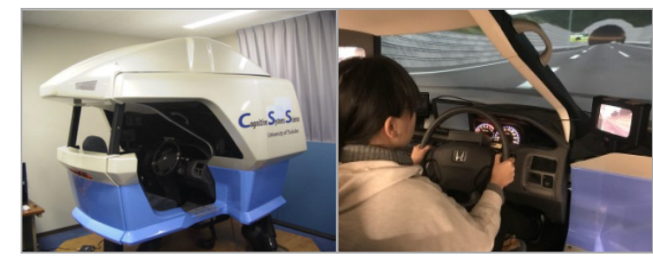

Figure 1. Simulator setup (HONDA, 2005)

\section{Experiment I}

The aim was to investigate how the interactions between the control authority and drivers' understanding of the system abilities combine to affect driver performance and safety. The steering function was automated to provide two different assistance systems for avoiding collisions with vehicles in the blind spot during a critical lane change (described below). Both systems were tested on three types of hazardous scenarios as shown in Figure 2.

Haptic Steering Control System (HSCS): This system provides haptic steering feedback by increasing the steering wheel friction torque from $1 \mathrm{~N} \cdot \mathrm{m}$ to $9.6 \mathrm{~N} \cdot \mathrm{m}$ to avoid collisions with vehicles in the blind spot during lane change. The increase in steering torque is accompanied by 
an auditory signal $(0.35 \mathrm{~s})$ to avoid surprising the driver. The system is deactivated when the hazard has been avoided, a collision occurs, or the driver overrides the additional steering torque to proceed with lane changing.

Automatic Steering Control Systems (ASCS): Using steer-by-wire function, this system decouples the steering input by the driver and automatically controls vehicle direction to avoid hazardous lane changes. When the system is activated, the driver may only control the vehicle speed. The system is deactivation when the hazard comes out of the system boundaries. The steering control transition between the driver and the system is guided by a set of auditory signals $(0.35 \mathrm{~s}$ each $)$ to inform the driver about system activation and deactivation.

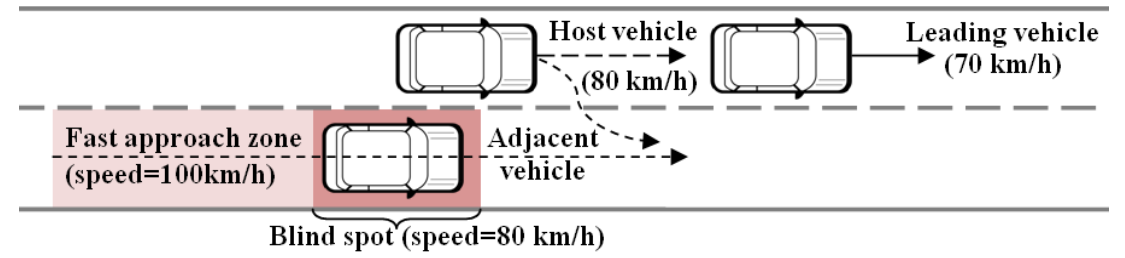

Figure 2. Hazardous lane change scenarios: \#1) Blind Spot Hazard: the adjacent vehicle is located in the blind spot of the host vehicle; \#2) Fast Approaching Hazard: the adjacent vehicle is a fast approaching car in the cruising lane wherein the system was not designed to detect it; and \#3) Combined Hazards: when a lane change manoeuvre is aborted by the driver or the system to avoid colliding with the adjacent vehicle in the blind spot of the host vehicle, the leading vehicle makes a sudden stop exposing drivers for the risk of rearend collision, which was undetectable for the system

\section{Experiment II}

The previous experiment does not consider the influence of drivers' ability to handle the situation. Automation systems may show different safety values under different degrees of risk, particularly when a visual limitation precludes an effective driver response. In this experiment, the ability of assistance systems to detect vehicles in the critical adjacent lane area was improved to a wider range than only the blind spot. The critical adjacent lane area is divided into four zones based on hazard position and drivers' ability to perceive the hazard as shown in Figure 3 .

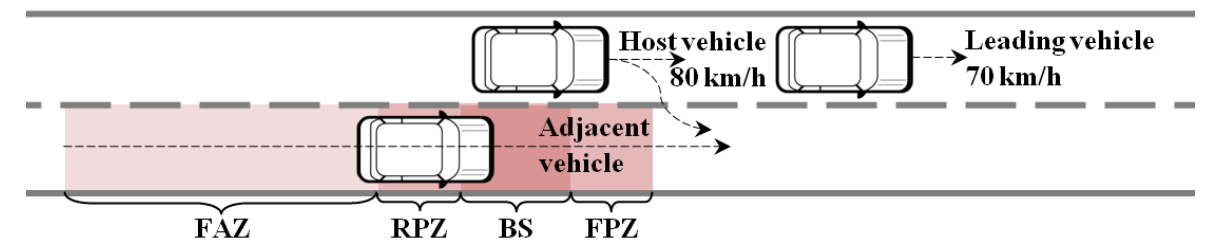

Figure 3. Hazardous lane change scenarios: \#1) front proximity zone (FPZ) in which the host driver can see the front part of the adjacent vehicle in the most right corner of the front window; \#2) rear proximity zone (RPZ) in which the rear part of the adjacent vehicle can be seen by the host driver in the right-hand mirror;

\#3) blind spot (BS) in which the host driver is not able to see the adjacent vehicle through the available screens; and \#4) fast approach zone (FAZ) in which the adjacent vehicle is a fast approaching car (approximately $100 \mathrm{~km} / \mathrm{h}$ ) with a time headway between 1.2 to $0.9 \mathrm{~s}$. at the time of lane change initiation

An adaptive collision avoidance system with a dynamic control allocation strategy depending on the situation is proposed. The design of the proposed system, hereinafter referred to as adaptive control system (ACS), integrates the characteristics of HSCS and ASCS. Based on the location of the hazard in the critical adjacent lane area, ACS sets off a warning and increases the steering wheel friction torque from normal $(1.5 \mathrm{~N} \cdot \mathrm{m})$ to $5 \mathrm{~N} \cdot \mathrm{m}, 7 \mathrm{~N} \cdot \mathrm{m}$, or $9.6 \mathrm{~N} \cdot \mathrm{m}$ when the hazard is located in FPZ, RPZ, or FAZ respectively. ACS provides automatic steering control assistance 
with haptic guidance $(4 \mathrm{~N} \cdot \mathrm{m})$ when the hazard is detected in the blind spot only. The design of HSCS and ASCS was improved to detect hazards in the critical adjacent lane area (in addition to the blind spot). The experiment followed a between subjects comparison considering four driving conditions: No automation assistance (NA), HSCS, ASCS, and ACS.

\section{Experiment III}

While the focus of Experiment II was to enhance system capabilities and design an effective and usable adaptive collision avoidance system (i.e., ACS) with a dynamic functional allocation, the aim of Experiment III was to improve the way the drivers interact with and adapt to the adaptive system using training-interaction approach. For each participant, the training was conducted in four days, once a week, during one month. In day \#1 (two hours), after receiving oral and written explanations and instructions, the drivers were introduced to the simulator and performed an appropriate number of familiarization (nonhazardous) drives on the simulator. The drivers then performed hazardous testing drives on highway setting using the four scenarios from experiment II without automation assistance (baseline). In day \#2 (one hour), the drivers performed the hazardous drives while supported by the adaptive collision avoidance system (ACS) after reading user manual of the system. In day \#3 and day \#4 (one hour each), the drivers first received intensive practical training on how to interact appropriately with the collision avoidance system before they encountered hazardous drives while supported by the system.

Table 1. Overview of the sample size and experimental design of each experiment

\begin{tabular}{|c|c|c|c|c|}
\hline Study & Date & Samples & Analysis & Automation Assistance \\
\hline \multirow[t]{2}{*}{ Experiment I } & \multirow{2}{*}{$\begin{array}{l}\text { May-July } \\
2016\end{array}$} & \multirow{2}{*}{$\begin{array}{c}\mathrm{n}=48(\lesssim 24, \text { ○ } 24) \\
M_{\text {age }}=30.0 ; S D_{\text {age }}=9.2\end{array}$} & \multirow{2}{*}{$\begin{array}{l}\text { Mixed factorial (2 groups ( } 24 \\
\text { drivers each) x } 3 \text { scenarios) }\end{array}$} & HSCS \\
\hline & & & & ASCS \\
\hline \multirow{4}{*}{ Experiment II } & \multirow{4}{*}{$\begin{array}{l}\text { Dec. } 2017- \\
\text { Feb. } 2018\end{array}$} & \multirow{4}{*}{$\begin{array}{c}\mathrm{n}=40(\text { え } 28, \text { ๆ } 12) \\
M_{\text {age }}=38.1, S D_{\text {age }}=10.8\end{array}$} & \multirow{4}{*}{$\begin{array}{l}\text { Mixed factorial (4 groups (10 } \\
\text { drivers each) x } 4 \text { scenarios) }\end{array}$} & No automation (NA) \\
\hline & & & & HSCS \\
\hline & & & & ASCS \\
\hline & & & & ASC \\
\hline Experiment III & $\begin{array}{l}\text { July-Oct. } \\
2018\end{array}$ & $\begin{array}{c}\mathrm{n}=42(\circlearrowleft 30,+12) \\
M_{\text {age }}=31.2, S D_{\mathrm{age}}=7.4\end{array}$ & $\begin{array}{l}\text { Within subjects (Between } \\
\text { experiment days comparison) }\end{array}$ & ASC \\
\hline
\end{tabular}

\section{RESULTS AND DISCUSSIONS}

The number of accidents for each type of assistance system was compared between driving experiments as shown in Table 2. The greatest number of collisions were observed in Experiment I under the HSCS and ASCS conditions when the drivers were most likely to misunderstand the system capabilities. The number of collisions was significantly reduced when improving system capabilities in Experiment II $\left(\chi^{2}(1)=13.6, p<0.05\right)$. The adaptive collision avoidance system (ACS) performed significantly better than the NA and HSCS conditions $\left(\chi^{2}(3)=47.4, p<0.01\right)$, but was less effective than ASCS. These results suggest an interaction between how drivers perceive the risk and interact with the system and the type of automation assistance. With regards Experiment III, which focused on improving driver-system interaction using training, Chi-square test showed that the number of collisions decreased significantly on day \#2 when driving with automation assistance compared to unsupported driving on day \#1 $\left(\chi^{2}(1)=125.8, p<0.01\right)$. The number of collision continued to fall on days \#3 and \#4. Furthermore, Experiment III resulted in significantly fewer collisions than Experiment II $\left(\chi^{2}(1)=133.8\right.$, 
$p<0.01)$. Both of these findings indicating the effectiveness of training in improving driver skills and system effectiveness.

Table 2. Number of collisions per driving condition for each experiment

\begin{tabular}{c|c|ccccc|cccc}
\cline { 2 - 12 } & \multicolumn{2}{c|}{ Experiment I } & \multicolumn{4}{c|}{ Experiment II } & \multicolumn{4}{c}{ Experiment III } \\
\cline { 2 - 14 } & HSCS & ASCS & NA & HSCS & ASCS & ACS & Day \#1 & Day \#2 & Day \#3 Day \#4 \\
\hline Number of Collisions & $39 / 96$ & $43 / 96$ & $27 / 40$ & $10 / 40$ & $0 / 40$ & $5 / 40$ & $77 / 168$ & $26 / 168$ & $8 / 168$ & $0 / 168$ \\
\hline
\end{tabular}

To evaluate how well drivers understand the assistance system, the maximum steering angle (MAS) during system activation was determined, as shown in Figure 4. For Experiment I, Tukey HSD and Bonferroni pairwise comparisons showed that the MSA under the ASCS condition was significantly larger than that under the HSCS condition $(\mathrm{p}<0.01)$. This suggests that drivers better understood the automation assistance when they were in charge of the steering control. The comparisons revealed significant differences between systems in Experiment II $(p<0.01)$. Consistent with the results of Experiment I, the lowest value of MSA was observed in the HSCS group. There were significant differences in MSA between Experiments I and II under conditions HSCS and ASCS $(p<0.05)$, which can be attributed to the further improvement of system capabilities in Experiment II compared to Experiment I. That is, in Experiment I, the drivers were exposed to hazards in which they were likely to overestimate the capabilities of the system, while the drivers in Experiment II were exposed to hazards that were within system design capabilities. Comparing this result with collisions data in Table 2 indicates that drivers' understanding only impacted on safety when the drivers were able to override the system intervention. In Experiment III, Tukey HSD and Bonferroni revealed significant effects of training interaction in reducing steering wheel angle during critical lane change maneuvers $(p<0.01)$. This indicates that with increased understanding of and adaptation to the system, drivers were able to improve how they interacted with the system. Thus, the further improvement in driver-system interaction achieved in day \#4 can be attributed to the training.
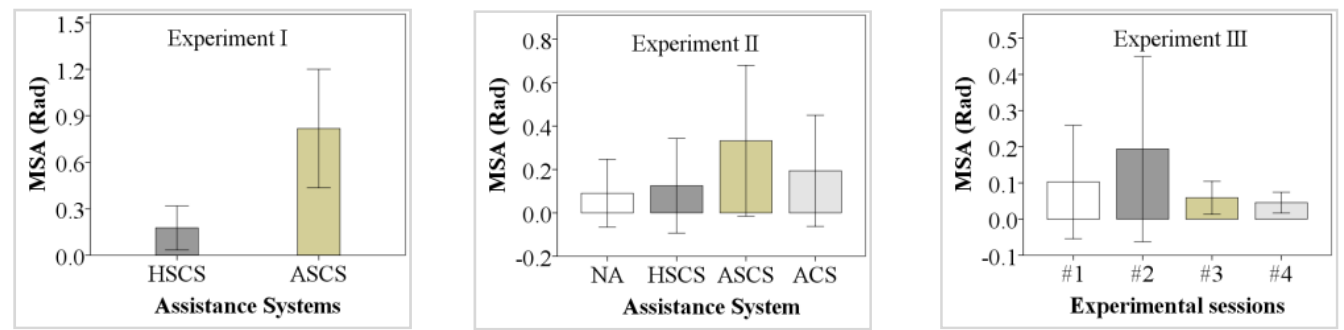

Figure 4. Mean and standard deviation of the maximum steering wheel angle during lane change manoeuvre

Knowing that in all experiments the drivers assumed the systems to be $100 \%$ reliable, these findings have important implications for the design of automation assistance. Implementing automation assistance without sufficient consideration of human factors, especially in unpredictable and highly dynamic traffic environment, can pose novel challenges for the drivers, such as automation-induced complacency as noted by (Parasuraman and Riley, 1997). Although the system capabilities were significantly improved in Experiment II compared to Experiment I, conflicts between drivers and automation assistance due to lack of drivers' understanding (mental model) of the system continued to occur result is accidents. Focusing on driver's skills and information processing abilities in Experiment III, driver-system interaction was significantly improved with the progress of training leading to enhance safety. 
Humans' understanding of automation can be related to their level of trust in the system (Abbink et al., 2012). On the one hand, humans may not be able to fully understand the assistance system until they can develop a certain level of trust in the system to reach their goal. On the other hand, the more humans understand the assistance system, the more they can develop an appropriate trust in the system. Thus, it was important to assess to what extent the drivers trusted the system. For the three driving experiments, Drivers' trust in the system was subjectively evaluated as shown in Figure 5. The Wilcoxon Rank Sum Test revealed a significant difference in trust level between systems in Experiment I $(\mathrm{Z}=-6.1, p<0.01)$ and in Experiment II $(\mathrm{Z}=-3.3, p<0.05)$. In Experiment I, the drivers rated their trust in HSCS slightly higher than ASCS. When compared with MSA results in Figure 4, drivers' trust in and understanding of the system were significantly correlated $(r=0.271, p<0.05)$. A similar pattern was found for Experiment II. In Experiment II, Drivers had significantly more trust in ACS than ASCS $(\mathrm{Z}=-4.2, p<0.05)$. Under the HSCS condition, drivers' steering behavior was smoother and more stable compared to ASCS and ACS (Figure 4), suggesting that drivers' trust in HSCS was more appropriate. Taken together and considering the number of collisions (Table 2), the ACS was, thus, able to strike a balance between the safety effectiveness, driver performance, and driver trust in automation.

Over the course of Experiment III, the drivers' level of trust in the system fluctuates before reaching on a more appropriate level. On day \#1, the drivers rated their trust in the system based only on expectations. On day $\# 2$, ratings of the system after the experiment showed a significantly reduced level of trust compared to day $\# 1(\mathrm{Z}=-6.3, p<0.01)$. Further interactions with the system on day \#3 led to an increased trust level. On day \#4, the training encouraged drivers to build an appropriate level of trust in the system. Although there was no significant difference in drivers' rating between days \#3 and \#4, the standard deviation of trust in day \#4 (S.D. $=0.09)$ indicates that the drivers rating of their feeling of trust was convergent. The significant improvement of system safety effectiveness on day \#4 indicates that drivers' trust in the system is more appropriate with the progress of training.
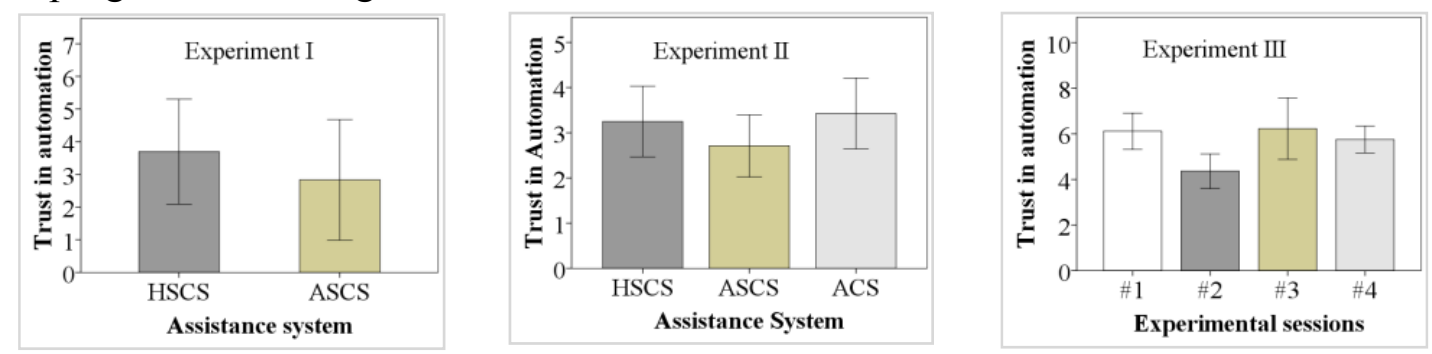

Figure 5. Mean and standard deviation of drivers' rating on their feeling of trust in the system

\section{CONCLUSIONS}

The present study attempts to reduce the gap between designers' point of view, which attempts to increase the level of automation and reduce the role of human and human factors point of view, which attempts to maintain the human as the main element of the system and make sure that the required physical and cognitive actions to engage in the automated function fall within human capabilities and limitations. The study conducted three driving experiments to assess how drivers supported by collision avoidance systems perform when exposed to various critical events. Experiments I indicated that overestimating the capabilities of the support system significantly degraded drivers' performance and the overall safety. Experiment II showed that the adaptive 
collision avoidance system which dynamically adjusted the capabilities of the system was able to address some of these limitations. Results also indicate that when expectations and system capabilities are aligned, drivers trust the system more appropriately and safety is improved. Experiment III showed that by training drivers how to interact with the systems further improved driver performance and safety.

These results indicate the importance of applying a human factors approach in the early stages of system design. While designing systems that take into the account human skills and abilities can go some way to improving their effectiveness, this alone is not sufficient. To maximize system safety and usability, it is also important to ensure that users understand its capabilities and limitations. For this, educating users how to operate the system is essential.

\section{ACKNOWLEDGMENT}

This work was supported by JSPS KAKENHI (15H05716).

\section{REFERENCES}

Abbink, D. A., Mulder, M., \& Boer, E. R. (2012). Haptic shared control: smoothly shifting control authority?. Cognition, Technology \& Work, 14(1), 19-28.

Carsten, O., Lai, F. C. H., Barnard, Y., Jamson, A. H., \& Merat, N. (2012). Control task substitution in semiautomated driving: does it matter what aspects are automated? Human Factors, 54(5), 747-761.

Hoc, J. M. (2000). From human-machine interaction to human-machine cooperation. Ergonomics, 43(7), 833-843.

Itoh, M., \& Inagaki, T. (2014). Design and evaluation of steering protection for avoiding collisions during a lane change. Ergonomics, 57(3), 361-373.

Kusano, K. D., \& Gabler, H. C. (2012). Safety Benefits of Forward Collision Warning, Brake Assist, and Autonomous Braking Systems in Rear-End Collisions. Intelligent Transportation Systems, IEEE Transactions on, 13(4), 1546-1555.

Muslim, H., \& Itoh, M. (2018). Effects of Human Understanding of Automation Abilities on Driver Performance and Acceptance of Lane Change Collision Avoidance Systems. IEEE Transactions on Intelligent Transportation Systems, 99, 1-11.

Muslim, H., \& Itoh, M. (2018). Steering Behavior with Different Levels of Automation Interventions for Avoiding Collisions During Lane Change. IEEE International Conference on Systems, Man, and Cybernetics, DOI: 10.1109/SMC.2018.00463, 2707-2712.

Muslim, H., \& Itoh, M. (2017). Human Factor Issues Associated with Lane Change Collision Avoidance Systems: Effects of Authority, Control, and Ability on Drivers' Performance and Situation Awareness. Proceedings of the Human Factors and Ergonomics Society Annual Meeting, 61(1), 1634-1638.

Parasuraman, R., \& Riley, V. (1997). Humans and Automation: Use, misuse, disuse, abuse. Human Factors, 39(2), 230-253.

Sheridan, T. B., \& Parasuraman, R. (2006). Human-automation interaction. Reviews of Human Factors and Ergonomics, 1, 89-129. 